\title{
Prevalence and potential risk factors of bovine fasciolosis in Gurage Zone, Abeshege district, Southern Ethiopia
}

\section{Research Article}

Volume: 2, Issue: 3 December 2018

Pages: 92-96

\section{Article History}

Received: 08.10.2018

Accepted: 13.11.2018

Available online:

14.11.2018

\section{Tegegn Dilbato $^{1^{*}}$ and Jemere Bekele ${ }^{2}$}

1.Dawuro Zone Tocha District Livestock and Fisheries Development Office, Dawuro, Ethiopia, Email: dilbatot@gmail.com. 2.Hawassa University School of Veterinary Medicine, Hawassa, Ethiopia, Email: jemerebekele@yahoo.com.

\begin{abstract}
A cross-sectional study aimed at estimating the prevalence and assessing the potential risk factors of bovine fasciolosis was carried out from November, 2011 to April, 2012 in Abeshege district of Gurage Zone. A total of 288 faecal samples were collected directly from the rectum of cattle and examination using sedimentation technique was performed. Overall $142(49.3 \%)$ cattle were found positive for faecal fluke egg detections. No significant variation was observed with feeding system, body condition, breed, sex, age, peasant association (PA) and herd size considered as potential risk factors $(P>0.05)$. However, water source was the only factor found to be significantly associated $(P<0.05)$ with occurrence of infections where cattle drinking water form rivers had $54.55 \%$ higher than those cattle getting water from other sources. The present study suggests that Fasciola infections in cattle is high and represent one of the constraints to livestock development in the study area. Hence, good management practice including provision of properly dried hay for those zero grazing cattle, an alternative use of lands of pasture, fluke elimination by regular treatment, and further detailed study could provide valuable information that foster local planning and implementation of a more sustainable fasciolosis control strategy for the district.
\end{abstract}

Keywords: Abeshege, bovine, fasciolosis, Gurage, prevalence, risk factors

DOI: $10.30704 /$ http-www-jivs-net.467948

To cite this article: Dilbato, T. \& Bekele, J. (2018). Prevalence and potential risk factors of bovine fasciolosis in Gurage Zone, Abeshege district, Southern Ethiopia. Journal of Istanbul Veterinary Sciences. 2(3), 92-96.

Abbreviated Title: J Ist Vet Sci

\section{Introduction}

Ethiopia has high livestock population, but productivity is very low as a result of disease, malnutrition and other management problems. Fasciolosis is one of major parasitic diseases contributing to loss in productivity (Abebe, 1992; FAO, 1993). Fasciolosis is caused by a leaf-shaped flukes whose anterior end is usually prolonged to the shape of cone. They are responsible for widespread morbidity and mortality in sheep and cattle characterized by weight loss, anemia and hypoproteinemia. The two most important species

*Corresponding Author: Tegegn Dilbato

E-mail: dilbatot@gmail.com are Fasciola hepatica which has worldwide distribution but mostly found in temperate and cooler areas of high altitude in the tropics and sub tropics while Fasciola gigantica is predominant in tropical areas including parts of Africa and Asia (Urquhart et al., 1996; Radostatis et al., 2007).

The prevalence of fasciolosis due to $F$. hepatica and F. gigantica in Ethiopia has long been known and its prevalence has been reported by Dejene (1987), Mitiku (1988) and Abebe (1990). Bovine fasciolosis is economically very important in that causing direct and indirect loss. The direct losses due to fasciolosis are

Journal homepage: www.jivs.net http://dergipark.gov.tr/http-www-jivs-net 
host mortality and liver condemnation, whereas indirect losses may occur in from of losses in body weight and reduced weight of lambs from infected ewes and decreased wool production, increased susceptibility to secondary infections and the expense of control measures (Jobre et al., 1998: Asrat et al., 2007).

The parasite lives part of its life in aquatic snails which acts as an intermediate host and found in and around wet areas such as waterholes and marshy. Farm animals are likely to pick up the infective stage of the parasite (metacercariae) if they drink from these sources (Okwole et al., 2000). Factors of stability are longevity of infection, chronic egg production of adult flukes (some 20000 egg or more/fluke /day) in sheep, availability of moisture (number of fluke larvae and snails a mean day and weight) temperature of at least $10 \mathrm{OC}$ is the factor of instability (Kassai, 1999).

No study and published reports are available in some parts of the country especially Gurage zone, Southern Nations, Nationalities and Peoples Regional State (SNNPRS) where the ecological conditions are likely to serve as favorable for the intermediate host and hence dissemination of infections to ruminants. Therefore, the main objective of this research is: To estimate the prevalence of fasciolosis in cattle in Abeshege district of Gurage zone. And to assess the potential risk factors associated with the occurrence of the disease.

\section{Materials and methods}

Description of study area: The study was conducted in Abeshege district of Gurage zone. Abeshege district is one of the 13 districts of Gurage zone, located in the Southern Nations Nationalities and People Region of Ethiopia, $150 \mathrm{~km}$ away from Addis Ababa at 080 17' $251^{\prime} \mathrm{N}$ latitude and $037046^{\prime} 023^{\prime \prime} \mathrm{E}$ longitude. Abeshege is bordered on the south by the Wabe River which separates it from Cheha district, on the west and north by the Oromia Region and on the east by Kebena district. The altitude of the district ranges from 1500-1850 meter above sea level with 75\% Woyna dega and $25 \%$ kola agro-ecological zones. The annual temperature and rainfall ranges are $11-220 \mathrm{C}$ and $800-1200 \mathrm{~mm}$, respectively. The society based on mixed farming system which include both crops and livestock production. The livestock population includes: 40.204 bovine, 4.217 equine, and 7.624 caprine, 1.825 ovine and 35.680 poultry. The most important food crops produced in the area are maize, sorghum, pepper, teff, haricot bean, and soybean (ADADO, 2004). The district (Abeshege) has 64,251 inhabitants ( 33.983 men and 30.268 women) with an area of $559 \mathrm{~km} 2$ with density of 114.9 inhabitants per km2 (ADADO, 2004).
Study animals: The study animals were different breed of cattle in Abeshege district. The animals in the study period were kept under different agroecologies, age groups and body conditions were recorded. The study involved 288 cattle from selected peasant associations (PA) of Abeshege district.

Study design: The cross- sectional study design type was used from November, 2011 to April, 2012 to estimate the prevalence and potential risk factors of bovine fasciolosis in Abeshege district.

Sample size and sampling procedures: Animals included in the study were selected with simple random sampling technique. The total number of cattle required for the research was calculated based on the formula given by Thrusfield (1995). For this study, $25 \%$ prevalence reported by Mulugeta (2008) in Hawassa was used to calculate the sample size using the following formula.

$\mathrm{N}=\frac{1.96^{2}(P e x)(1-P e x)}{d^{2}}$

Where, $\mathrm{N}=$ Sample size

Pex $=$ Expected prevalence

$d=$ Desired level of precision (5\%)

$1.96=$ Value of $Z$ at $95 \%$ confidence interval

Then, $N=\frac{1.96^{2}(0.25)(1-0.25)}{(0.05)^{2}}=288$

The expected prevalence of fasciolosis in Hawassa was 25\% (Mulugeta. 2008).

\section{Study methodology:}

Coprological examination: Faecal samples were collected directly from the rectum of randomly selected animals and examination carried out in the field as much as possible. The samples were collected in tightly closed universal bottles and examined for presence of Fasciola eggs. Faecal examinations for fluke eggs usually require use of faecal sedimentation (Kassai, 1999). Accordingly, a total of 288 faecal samples were collected and processed.

Sedimentation procedures concentrate both eggs and faeces at the bottom of a liquid medium (water) and help to detect fluke eggs that have too high specific gravity (Hendrix, 1998). Therefore, the samples were analyzed using sedimentation procedure to demonstrate liver fluke eggs which are large, golden, and yellow in color. Briefly, about 3 grams of faeces as weighed and $42 \mathrm{ml}$ of tap water was poured to it. It was thoroughly mixed with a stirring device. The suspension was then filtered through a tea strainer. The filtered suspension was poured in to test tube which stood in the rack. The test tubes with filtered material was put in centrifuge 
and centrifuged for three minutes for $1200 \mathrm{rpm}$. The supernatant was carefully discarded after centrifuged. The resulting sediment was stained by adding two drops of methylene blue and shacked carefully. A small drop of the stained sediment was transferred to a microscope slide using a pipette. Cover slip was put to over the droplet. It was then examined under microscope at $10 \mathrm{x}$ objective.

Data on hypothesized risk factors like epidemiological area (peasant associations), breed, sex, age, body condition, watering source, feeding system and herd size of the study cattle were recorded. These data were collected by asking the owner on feeding system, water source, herd size and age of the animal and body condition. By visual examination the breed and sex of animal were registered as being local and cross breed: and male and female. According to Dabas et al., (2007), the age was categorized in to calf (0-1 year), yearling (1-3 year), and adults ( $>3$ year) based on information from the owner, dentition and observation of the horn of study animals.

Data management and analysis: The findings from coprological examinations and the epidemiological data were recorded in Microsoft Excel spreadsheet and all analysis was conducted using STATA 11 . Chi-square $(x 2)$ test was used to assess the association between prevalence and associated risk factors. In all cases of the analysis the confidence interval was set at $95 \%$ and $P$ values less than 0.05 was considered significant.

\section{Results and Discussion}

Prevalence and Potential risk factors: Out of the total 288 faecal samples examined, 142 (49.3\%) were found positive for Fasciola eggs. There was no significant variation ( $x 2=0.63$ : $P>0.05)$ observed in prevalence between the different (PAs) found in the study district as the prevalence only ranged from $46.43 \%$ at Laygnaw Garaba to $52.12 \%$ in Ambelta (Table 1).

Table 1. Prevalence of bovine fasciolosis in different Kebele of Abeshege district.

\begin{tabular}{lccc}
\hline Kebele & Examined & Positive & Prevalence (\%) \\
\hline Ambelta & 46 & 24 & 52.12 \\
Mammedie & 58 & 29 & 50 \\
Laygnaw Garaba & 56 & 26 & 46.43 \\
Tachegnaw Garaba & 62 & 29 & 46.77 \\
Tatesa & 66 & 34 & 51.5 \\
Total & 288 & 142 & 49.3 \\
\hline
\end{tabular}

$X^{2}=0.63 ; P=0.96$
Moreover, there was no significant variation $(P>0.05)$ observed in terms of breed, sex and age of animals basis of faecal egg findings (Table 2).

Table 2. Prevalence of bovine fasciolosis based on breed, sex and age as risk factors.

\begin{tabular}{|c|c|c|c|c|c|c|}
\hline \multicolumn{2}{|c|}{ Risk Factors } & \multirow{2}{*}{$\begin{array}{c}\text { Examined } \\
182\end{array}$} & \multirow{2}{*}{$\begin{array}{c}\text { Positive } \\
91\end{array}$} & \multirow{2}{*}{$\begin{array}{c}\text { Prevalence } \\
50\end{array}$} & \multirow{2}{*}{$\frac{x^{2}}{0.09}$} & \multirow{2}{*}{$\frac{\boldsymbol{P} \text {-value }}{0.75}$} \\
\hline Breed & Local & & & & & \\
\hline & Cross & 106 & 51 & 48.11 & & \\
\hline & Total & 288 & 142 & 49.3 & & \\
\hline \multirow{4}{*}{ Sex } & Male & 119 & 59 & 49.58 & \multirow{4}{*}{0.006} & \multirow{3}{*}{0.94} \\
\hline & Female & 169 & 83 & 49.11 & & \\
\hline & Total & 288 & 142 & 49.3 & & \\
\hline & Calves & 28 & 11 & 39.3 & & \\
\hline \multirow[t]{3}{*}{ Age } & Young & 87 & 43 & 49.4 & 1.29 & 0.52 \\
\hline & Adult & 173 & 88 & 50.86 & & \\
\hline & Total & 288 & 142 & 49.3 & & \\
\hline
\end{tabular}

And again there was no significant variation $(P>0.05)$ revealed in terms body, feeding and herd size whereas significant difference was noted with regard to water source (Table 3).

Table 3. Prevalence of bovine fasciolosis based on body condition, feeding, herd size and water source.

\begin{tabular}{lccccc}
\hline Risk factors & Examined & Positive & Prevalence & $\mathbf{X}^{2}$ & $P$-value \\
\hline Body Condition & & & & & \\
Poor & 45 & 27 & 60 & & \\
Medium & 99 & 47 & 47.47 & 2.44 & 0.295 \\
Good & 144 & 68 & 47.22 & & \\
Total & 288 & 142 & 49.3 & & \\
Feeding & & & & & \\
Grazing & 206 & 109 & 52.91 & & \\
Zero grazing & 37 & 15 & 40.54 & 3.76 & 0.15 \\
Tethering & 45 & 18 & 40 & & \\
Total & 288 & 142 & 49.3 & & \\
Water source & & & & & \\
River & 187 & 102 & 54.55 & 13.61 & 0.001 \\
Pond & 61 & 31 & 50.82 & & \\
Pipe & 40 & 9 & 22.50 & & \\
Total & 288 & 142 & 49.3 & & \\
Herd size & & & & & \\
$<10$ & 48 & 20 & 41.67 & & \\
$10-30$ & 101 & 46 & 45.54 & 3.29 & 0.192 \\
$>30$ & 139 & 76 & 54.68 & & \\
Total & 288 & 142 & 49.3 & & \\
\hline
\end{tabular}

The overall prevalence of bovine fasciolosis using coprological examination in the current study area is $49.3 \%$. This finding shows a lower prevalence as compared to the results of other workers in other parts of the country such as, $80-89 \%$ in Debre Berhan (Dagne, 1994) cited in (Yilma and Mesfin, 2000), 82.5\% in Western Shewa (Beyene, 1994), and moderately 
higher as compared to $25 \%$ by Mulugeta (2008) in Hawassa, $28 \%$ recorded by Melaku (1991) in Chilalo. However, this finding was comparable to the prevalence of $53.72 \%$ reported by Abebe (1990) at Arsi administrative region. The differences observed between this study and the other works might be due to differences in ecological and climatic conditions which may vary from place to place and also within the same area from year to year.

The prevalence of fasciolosis in the district only varied from $46.43 \%$ in Laygnaw Garaba to $52.12 \%$ in Ambelta (PA). Such little ranges between PAs emerged to reveal no significant variations between them in detections of faecal eggs. This might be due to little variation in the ecological conditions in the district as well as access of metacercariae to cattle of all PA with the pasture harvested from the border of the rivers and marshy areas and/ or by grazing or watering in the shore of rivers as well as marshy areas, There was no significant variation between local and cross bred cattle that the prevalence in local breed was $50.0 \%$ while $48.11 \%$ in cross breed cattle. This might be due to an access of gaining metacercariae to both breed equally in either grazing or zero grazing with hay having sufficient moisture content for the survival of metacercariae to infect animals.

In the present study, there was no statistically significant association $(P>0.05)$ in prevalence between female (49.11\%) and male (57.98\%) cattle. This finding showed that both sexes are equally susceptible and exposed to the infection and this might be due to grazing of both sexes in metacercariae contaminated pasture and swampy or marshy area. The current work indicated that there was no statistically significant association between different age groups of the study animals with prevalence of fasciolosis. It was $39.3 \%$ in calves, $49.4 \%$ yearlings and $50.86 \%$ in adults. Since most of the time cattle of all ages access the same pasture zones, infection is picked up without regard to age with young cattle getting infected almost immediately they are introduced to full grazing (Phiri et al., 2005).

Our findings also showed that there was no statistically significant difference $(P>0.05)$ in prevalence among different body condition groups of study animals. It was $60 \%$ in poor, $47.47 \%$ in medium and $47.22 \%$ in good body condition animals. The prevalence was moderately high in poor body condition animals and similar in medium and good body condition animals. This might be due to animals with chronic liver fluke disease are often in poor body condition (Mc Gavin and Zachary, 2007).
In the present study, there was no significant association between prevalence and the feeding system of the study animals. This might be due to lack of wide ranging disparity among the feeding system in terms of ecological factors and difference in microclimate for the snail intermediate host and the parasite Fasciola. In the same way, presence of annual over flooding during the rainy season leaving pockets of water bodies, presence of marshy area for long period during the dry season, slowly river and swampy parts of the lakes and the river borders are contributory. It is a clear practice that people in this area usually graze their animals, particularly cattle, in swampy area and similar ecosystem as there is shortage of pasture mainly during drier months of the year. Such ecological conditions are considered favourable for breeding and survival of the intermediate host snail and the parasite (Urquhart et al., 1996).

Statistical analysis indicated that there was significant difference between prevalence and different water source for study animals $(P<0.05)$. The high prevalence (56.04\%) was recorded in those cattle drinking water from the river and this might be due to the chance of getting metacercariae during grazing on the border of the river and drinking slowly flowing river. The prevalence was low $(22.5 \%)$ in cattle getting water from the pipe. In this case the source of metacercariae could only be from feed such as hay harvested from swampy areas having sufficient moisture content for survival of metacercariae. Furthermore, there was no significant variation among the herd size $(P>0.05)$. The reason might be due to lack of ecological variability though it was anticipated that concentration of animals in one place facilitates the development of the parasite. The concentration (high herd size) is conductive to heavy contamination and distribution of the herd (low herd size) over a large area, decreased probability of infection, especially in herd grazing around a water hole (Fischer and Say, 1989).

\section{Conclusion and Recommendations}

The present study demonstrated that bovine fasciolosis is common in Abeshege district of Gurage zone, Southern Nations, Nationalities and People regional State (SNNPRS). No variability between the potential risk factors is suggestive of favorable environmental conditions with little range of variation. Hence, it would be essential to exercise the following strategies to alleviate the problem in the current area.

Create awareness among farmers about the disease to enable them to avoid local practices which 
could expose their animals to infection and to help them identify animals suffering from the disease and present them to treatment.

Strategic use of anthelmintic combined with improved pasture management.

Zero grazing animals should be provided with dried hay rather than hay having sufficient moisture for the survival of metacercariae.

Comprehensive study should be carried out involving year round schedule and Gurage zone in general to determine the factors which contribute to the high prevalence of bovine fasciolosis in the area and information used to develop control strategies.

\section{References}

Abebe, R. (1992). Fasciolosis Clinical occurrence, Coprological abattoir and snail survey in around Wolliso, DVM thesis, Addis Ababa University, Faculty of Veterinary Medicine Debre Zeit, Ethiopia.

Abebe, W. (1990). Prevalence of Bovine Fasciolosis in Arsi Administrative Region. DVM thesis, Addis Ababa University. Faculty of Veterinary Medicine, Debre Zeit, Ethiopia.

ADADO, Abeshege District Agriculture Development Office, ( 2004). Unpublished socio-economic data.

Asrat, M., Peden, D., Jobre, Y., Girma, Tadesse, G., Abebe, G., \& Gideyelew, T. (2007). Evaluation of Spatial distribution of F. hepatica and F. gigantica using geographic information system (GIS) in the Nile River Basin. Ethiopian Veterinary Journal, 11(2), 41-57.

Beyene, Y. (1994). Epidemiology of Bovine and Ovine Fasciolosis and Distribution of its Snail Intermediates Host in Western Shewa, DVM thesis, Addis Ababa University. Faculty of Veterinary Medicine Debre Zeit, Ethiopia.

Dabas, V. P., Saxena, O. P. \& Dabas, R. (2007). Veterinary jurisprudence and postmortem, 3rd ed. IBDC (International) Book Distributing Co.

Dagne, M. (1994). Survey on Prevalence and Economic Significance of Bovine Fasciolosis in Debre Berhan Region, DVM thesis, Faculty of Veterinary Medicine, Addis Ababa University, Debre Zeit, Ethiopia.

Dejene, G. (1987). Incidence and Economic Significance of Fasciolosis in Wolaita Awuraja, Sidama, Ethiopia DVM thesis Addis Ababa University Faculty of Veterinary Medicine, Debre Zeit, Ethiopia.

FAO, (1993). Ethiopia livestock sector development project Preparation report volume 1, FAO. Rome Italy. 51.

Fischer, M. S. \& Say, R. (1989). Manual Tropical Veterinary Parasitological UK: CAB International.

Hendrix, C. M. (1998). Diagnostic Veterinary Parasitology 2nd ed. Auburn University Alabama: Mosby.

Jobre, Y. M., Malone, J. B., Gommes, R., Hansen, J., Shinegbrg, J., Snijders, F., Nachet, O. F. \& Ataman, E. (1998). A geographic information system on the potential distribution and abundance of F. hepatica and F. gigantica in East Africa based on Food Agriculture Organization data

\section{Acknowledgments}

Our deepest gratitude is extending to Wolaita Soddo Regional Veterinary Laboratory Parasitological Department experts especially, Mr. Tekle Alaro and Mrs. Mulualem Zaza for their help in the field as well as in the laboratory activities.

bases. Veterinary Parasitology 78, 87-102.

Kassai, T. (1999). Veterinary Helminthology 1st ed. Development of Parasitology and Zoology, University of Veterinary Science. Budapest: Hungary.

McGavin, M. D. \& Zachary, J. F. (2007). Pathologic basis of veterinary disease: 4th ed. Phledelphia, US: Mosby Elsevier.

Melaku, Z. (1991). Prevalence of Ovine Gastrointestinal Helminthes of cattle Chilalo Awuraja. Arsi Admistrative Region. DVM thesis, Addis Ababa University, Faculty of Veterinary Medicine, Debre Zeit, Ethiopia.

Mitiku, A. (1988). Prevalence of Bovine Fasciolosis and its Economic Significance at Nekemite. DVM thesis, Faculty of Veterinary Medicine, Addis Ababa University, Debre Zeit, Ethiopia.

Mulugeta, B. H. (2008). Prevalence of Fasciola in Hawassa determined on the basis of Abattoir examination. DVM thesis, Faculty of Veterinary Medicine, Jimma, Ethiopia.

Okewole, E. A., Ogundipe, G. A. T., Adejinmi, J. O. \& Olaniyan, A. O. (2000). Clinical Evaluation of the three chemoprophylactics regimes against ovine heliminthosis in a fasciola endemic farm in Ibadan, Nigeria. Israel Journal of Veterinary Medicine, 56(1), 15-28.

Phiri, A. M, Phiri, I. K., Siziya, S., Sikasunge, C. S., Chembensofu, M., \& Monrad, J. (2005). Seasonal Pattern of Bovine Fasciolosis in the Kafue and Zambezi catchment areas of Zambia. Zambia, University of Zambia. School of Veterinary Medicine. Department of Clinical Studies. Lusaka. Zambia. Veterinary Parasitology, 134(1-2), 87-92.

Radostatis, O. M., Gay, C. C., Hichcliff, K. W. \& Constable, P. O. (2007). Veterinary medicine. A Text book of the diagnosis of cattle, horses, sheep, pigs. 10th ed .London, UK: Saunders Ltd.

Thrusfield, M. (1995). Veterinary Epidemiology. Department of Veterinary Clinical Study. 2nd ed. Ames, lowa, US: Blackwell Science.

Urquhart, G. M., Armour, J., Duncan, J. L., Dunn, A. M., Jennings, F. W. (1996). Veterinary Parasitology. 2nd ed. Scotland, UK: Blackwell Science.

Yilma, J. M. \& Mesfin, A. (2000). Dry season bovine fasciolosis in Northwestern part of Ethiopia. Revue de Médecine Vétérinaire, 151(6), 493-500. 